\title{
Predicting the mechanical behaviour of large composite rocket motor cases
}

\author{
N. Couroneau \\ DGA/CAEPE, St Médard en Jalles, France
}

\begin{abstract}
A method to develop finite element models of the rocket motor cases of a strategic missile is presented. Based on the use of multivariate analyses, this approach is made necessary given the impossibility to control all input data: difficult characterization of filament wound materials, influence of manufacturing and qualification processes, unknown fabrication parameters, etc. An initial reference model is built up using preliminary data and theoretical winding laws. Having compared the models predictions and available structural test results, a sensitivity analysis is carried out to discuss the individual influence of the input data on the accuracy of the predictions. The multivariate analysis finally enables a global assessment of the parametric analysis results.

Keywords: rocket motor case, composite filament winding, finite element model, variable scattering, multivariate analysis, test prediction.
\end{abstract}

\section{Introduction}

In addition to providing ground test facilities for the development of the future generation of French strategic missiles, the Centre d'Achèvement et d'Essais des Propulseurs et Engins (CAEPE) is responsible for evaluating the degree of performance, durability and security of the solid propellant rocket motors constituting the missiles. The mechanical expertise work is carried out using finite element models able to predict the stresses and strains of the mechanical parts throughout the fabrication and service life.

Many difficulties arise when attempting to describe the mechanical behaviour of the rocket motor cases. The filament-wound composite structures have complex geometry and properties especially in the dome area. As the wound layers are added on a cylindrical mandrel, the curvilinear path leads to a 
continuous change in the winding angle and thickness. In addition, the fiber angle varies in the thickness direction because the fiber path depends on the surface on which fibers are wound. Other concerns are due to the difficult characterization of the different materials and the variability of their properties.

The method chosen here is to take advantage of various complex tests performed on the full scale specimen in order to identify uncertain material properties or geometrical parameters. This approach, known as the experimental / numerical dialog or inverse identification [1], is presented here for the two largest composite structures of a strategic missile.

\section{Development of the initial model}

\subsection{Architecture}

The solid propellant rocket motors corresponding to the two first propulsion stages of the French strategic missiles share a common outer diameter $(>2 \mathrm{~m})$. Their structure is based on the same architecture and the same materials:

- The carbon-epoxy filament-wound pressure vessel, acting both as the propellant tank and the combustion chamber,

- The two metal polar mountings located at the aft and front openings to provide connection with the igniter and the nozzle,

- The two cylindrical skirts, made of both carbon-epoxy tissues and circumferential windings, ensuring the connection with the rest of the carrier,

- Rubber connections between the vessel and the skirts on the one hand, and between the vessel and the polar mountings on the other hand.

\subsection{Winding law}

The composite envelope is constituted of a succession of circumferential windings on the cylindrical part and satellite windings running between the two openings. The laminate lay-up in the cylindrical area is rather simple to describe with an assumed constant value of winding angle and direction of all plies. Conversely, the complex dome geometry involves a rapid change in angle and thickness along a meridian. Different netting theories [2] based on geometrical or mechanical approaches describe this distribution. The planar theory used here assumes that the fiber patterns lie in a plane which is tangent to the polar opening at one end and tangent to the opposite side of the polar opening at the other end.

The winding angle calculated (Fig. 3a) with the planar theory at a given location is applied as a constant value to all plies. The thickness distribution (Fig. 3a) shows good agreement with the measured values (Fig. 3b). A preliminary analysis was performed to investigate the effect of an evolution of the winding angle in the thickness direction, as suggested by Park et al [3]. The thickness of the first ply was calculated from the mandrel shape whereas the subsequent plies were calculated with the updated shape. The difference in angle between plies was particularly noticeable near the polar bosses where the 
thickness is more important. However this induced no significant change in the overall calculated displacements.

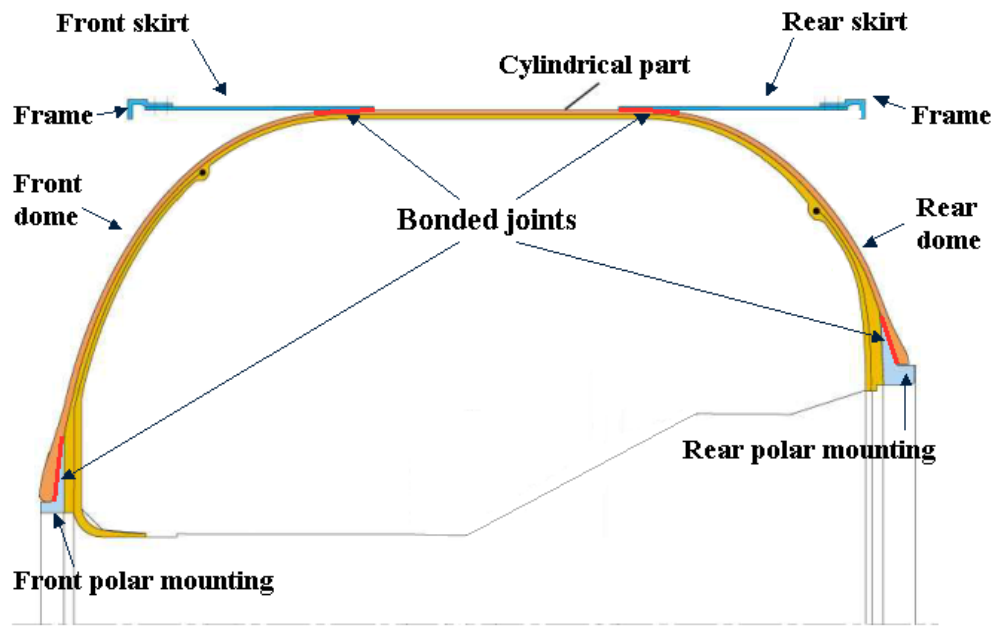

Figure 1: Typical components of a rocket motor case (half-view in section).

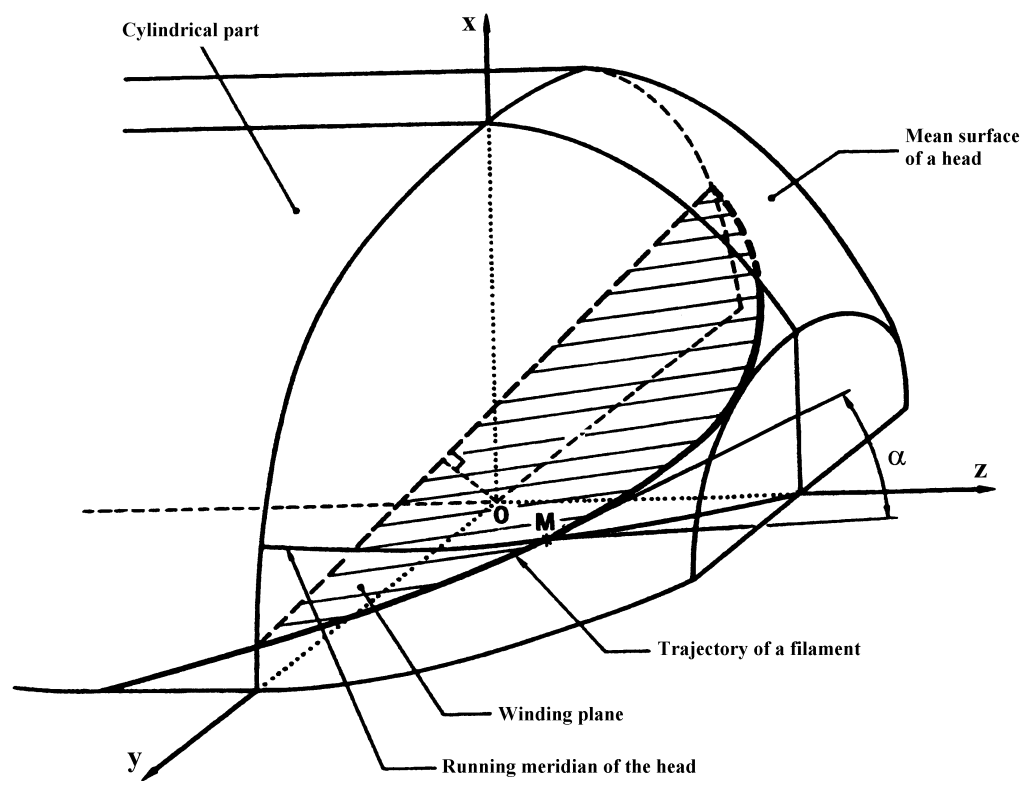

Figure 2: Winding path. 


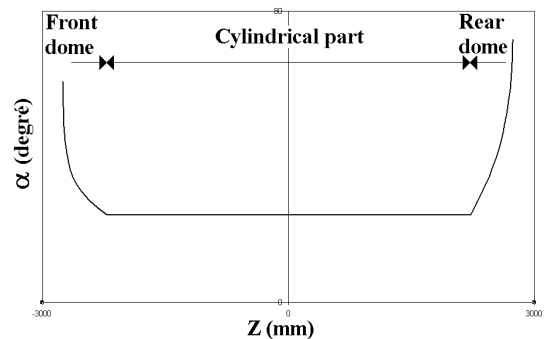

a) Winding angle

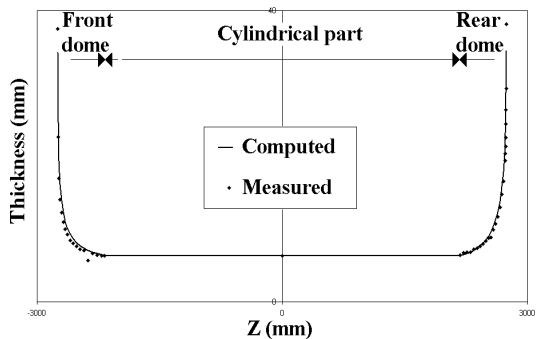

b) Thickness

Figure 3: $\quad$ Planar winding law applied to the first stage.

\subsection{Finite element models}

The finite element analyses considering the geometrical non-linearities are performed using ANSYS code. The initial axi-symmetric models are developed over a small angle in order to use the composite dedicated 8-node finite elements, allowing for a direct input of the materials lay-up and fibers orientation. The data are transmitted to Ansys by means of a Fortran routine incorporating geometric parameters, materials properties and winding laws. A larger density of elements is used near the openings where the winding angle and thickness vary abruptly.

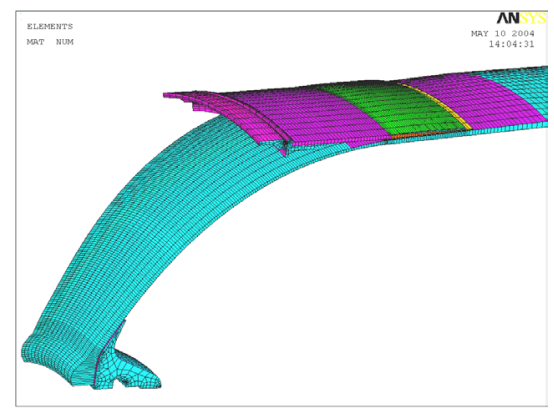

a) First stage

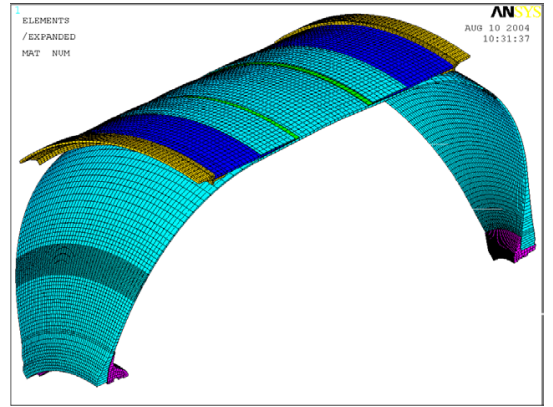

b) Second stage

Figure 4: $\quad 3 D$ mesh using solid elements.

The model contains general boundary conditions for axisymmetry and full displacement constraints on the frames to take account of the inter-stage skirts which are not included in the models.

\subsection{Evaluation of the models}

Two load cases are selected to evaluate the finite element model performance : internal pressure (Fig. 5a) and axial compression (Fig. 5b). The internal pressure load case is corresponding to the pressure proof test carried out to accept the 
structure prior to its filling up with propellant. It is conducted with an internal pressure $15 \%$ higher than the operating pressure and an axial load applied on the aft polar mounting to represent the thrust loads. The axial compression load case is applied on the rear frame with the front frame being blocked. It is representative of an aggression of the missile when stored in the submarine and it also represents the load transmitted to the second stage during the first stage flight. These two tests are carried out and analysed by the contractor in charge of the rocket motor cases design and fabrication.

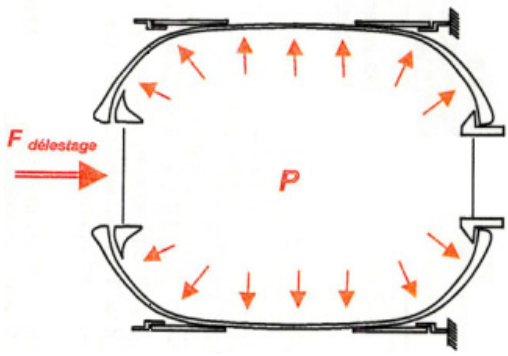

a) Internal pressure

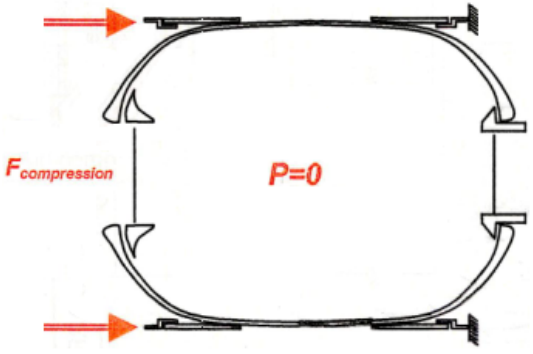

b) Axial compression

Figure 5: Load cases for evaluating the models.

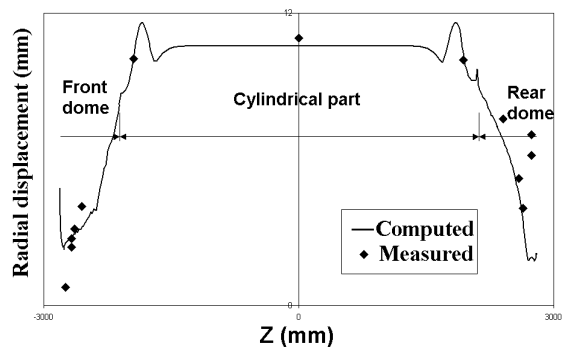

a) First stage

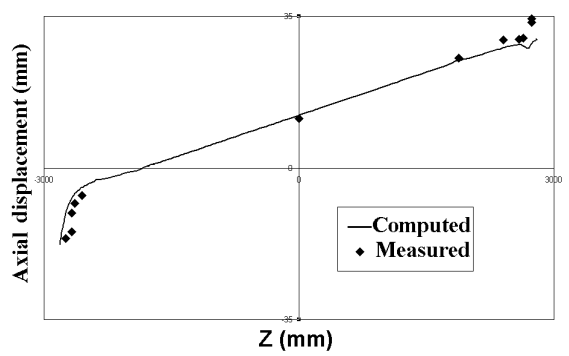

c) First stage

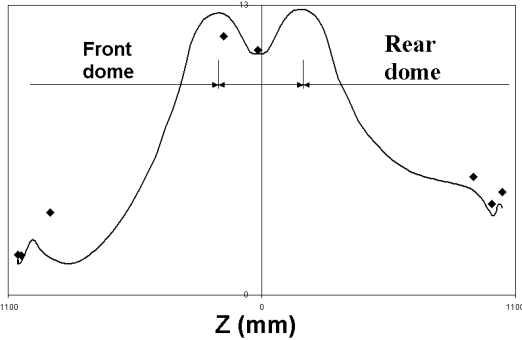

b) Second stage

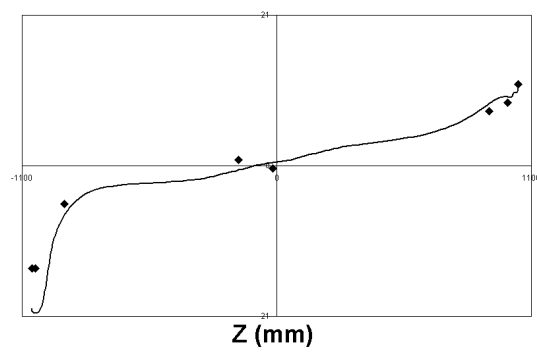

d) Second stage

Figure 6: Model evaluation for the internal pressure load case. 
The results obtained for the internal pressure load case are shown in Figure 6. The computed and measured displacements are plotted all along a meridian of the composite vessel. This comparison shows a good agreement except in the regions near the polar openings at the rear and front. The average difference is about $1.5 \mathrm{~mm}$ for the $1^{\text {st }}$ stage and $1.1 \mathrm{~mm}$ for the $2^{\text {nd }}$ stage, with maximum values of 5 to $6 \mathrm{~mm}$.

For the axial compression load case, only the axial displacements of the skirts and the cylindrical part of the structure are used, the other values being too small for comparison. No experimental results are available here so the contractor's calculations are used instead. The calculations are all in good agreement with an average difference of about $0.2-0.3 \mathrm{~mm}$ for the two models.

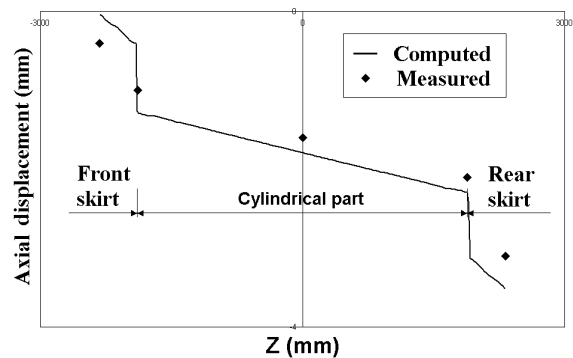

a) First stage

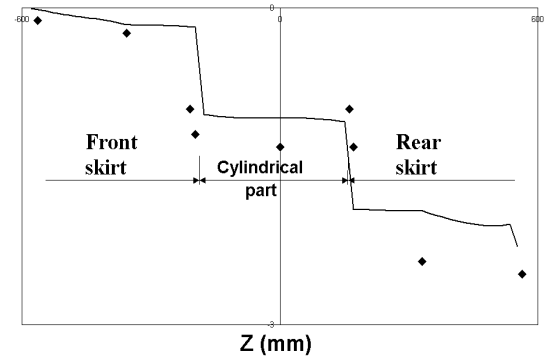

b) Second stage

Figure 7: $\quad$ Model evaluation for the axial compression load case.

\section{Optimization of the initial model}

\subsection{Parameters influence analysis}

An analysis is conducted to identify the influence of each parameter considered individually on the response of the model and the difference between calculations and experiments. Only the internal pressure proof test is considered here as there are no experimental data for this load case and the difference with the contractor calculations is very satisfactory.

The main reasons for discussing the accuracy of the input data of the model are listed below:

1) Mechanical properties of composite materials : lack of representativity of the characterization tests performed on uni-directionnal laminate plates with respect to the actual fabrication process of the structures, variability of materials properties and possible damage of the matrix and fibers after the pressure proof test [4],

2) Winding law : Unknown fabrication parameters, theoretical law assumed without possibility of a direct validation, expected slippage of the fiber during the winding process,

3) Initial geometry: Possible evolution of the initial geometry during subsequent fabrication stages [5] or after the pressure proof test. 
The different parameters above are modified one by one in the reference model (Tab. 1). For each of them, a possible variation is assumed and each case results in a new computation being performed. The matrix degradation is modelled by a decrease of the transverse modulus of the hoop layers, the winding laws are transformed by an offset or a multiplying coefficient, and the initial geometry is modified by means of a prior pressurization of the model.

Table 1: $\quad$ Description of the sensitivity analysis.

\begin{tabular}{|c|c|c|}
\hline \multirow{4}{*}{ Materials properties } & \multirow{3}{*}{ Corlposite structure } & $\mathrm{E}_{\mathrm{L}}=\mathrm{E}_{\mathrm{L}}$ reference $+i-5 \%$ \\
\hline & & $E T=E T$ reference $+/ 20 \%$ \\
\hline & & ET $=$ ET reference $-50 \%$ and $-75 \%$ (thodelling mattix degradation) \\
\hline & Skirts & $\mathrm{ET}=\mathrm{EC}=\mathrm{E}$ reference $+r-20 \%$ \\
\hline \multirow{5}{*}{ Winding law } & Cylindrical part & $\alpha_{0}=\phi_{0}$ reference $+\lambda_{-1}{ }^{\circ}$ \\
\hline & \multirow{2}{*}{ Front dome } & Translation of $+f-10^{\circ}$ \\
\hline & & MLaltiplication by $+r-10 \%$ \\
\hline & \multirow{2}{*}{ Reat dothe } & Translation of $+i-10^{\circ}$ \\
\hline & & Multiplication by $+r-10 \%$ \\
\hline Itritial geometty & Frior deformation & $F_{\text {irit }}=10$ and 20 bar \\
\hline
\end{tabular}

The results of the sensitivity analysis are summarized in Table 2. The modifications leading to an improvement of the results are indicated in bold characters whereas those leading to a deterioration are printed in italics. The major factors of influence given the chosen variations of the different parameters are the structure moduli, the changes applied to the winding laws and the prior pressure deformation.

The calculations related to a change in the materials properties tend to indicate an overvaluation of the reference values or a possible matrix degradation. Concerning the winding laws, the results show a significant latitude for improving the initial theoretical laws. The 10 bar prior pressure deformation of the $2^{\text {nd }}$ stage model also results in an important decrease of the difference between calculations and measurements.

\subsection{Multivariate analysis}

The multivariate analysis performed using Matlab allows for a global assessment of the results of the parameters influence analysis. The data processing consists of a linear system to be solved using the least square method as per eqn (1). The output data is the best combination of modified parameters to minimize the difference between experiments and computations :

$$
\text { [A]. }\{\mathrm{x}\}=\{\mathrm{b}\}
$$

where $[\mathrm{A}]$ is a matrix containing the results of parameters influence analysis, $\{\mathrm{x}\}$ is the requested solution,

$\{b\}$ contains for each of the measurement points the difference between experimental and numerical values of displacements. 
Table 2: $\quad$ Results of the parameters influence analysis.

\begin{tabular}{|c|c|c|c|c|c|c|c|c|c|}
\hline & \multicolumn{4}{|c|}{1 st stage } & \multicolumn{4}{|c|}{ 2nd stage } \\
\hline & & \multicolumn{2}{|c|}{ Radial error (mm) } & \multicolumn{2}{|c|}{ Axial error (mm) } & \multicolumn{2}{|c|}{ Radial error (mm) } & \multicolumn{2}{|c|}{ Axial error (mm) } \\
\hline & & Mean & Maximum & Mean & Maximum & Mean & Maximum & Mean & Maximum \\
\hline \multicolumn{2}{|c|}{ Reference } & 1,4 & 5,1 & 1,7 & 4,6 & 0,8 & 2,1 & 1,4 & 6,2 \\
\hline \multirow{6}{*}{ 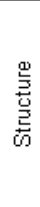 } & $E_{L}+5 \%$ & 1,5 & 5,2 & 2,2 & 59 & 0,9 & 2,3 & 1,4 & 5,6 \\
\hline & $E_{L}-5 \%$ & 1,4 & 5,0 & 1,3 & 3,1 & 0,7 & 2,0 & 1,5 & 0,0 \\
\hline & $\mathrm{E}_{\mathrm{T}}+20 \%$ & 1,5 & 5.1 & 2,0 & 5,0 & 0,9 & 2,3 & 1,5 & 6,6 \\
\hline & $E_{T}-20 \%$ & 1,4 & 5.1 & 1,4 & 4,2 & 0,8 & 2,1 & 1,4 & 5,8 \\
\hline & $E_{T}-50 \%$ & 1,3 & 5,1 & 1,2 & 3,5 & 0,0 & 1,0 & 1,2 & 5,0 \\
\hline & $\mathrm{E}_{\mathrm{T}-75 \%}$ & 1,2 & 5,2 & 1,3 & 2,9 & 0,7 & 1,6 & 1,0 & 4,4 \\
\hline \multirow{2}{*}{ 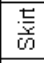 } & $E+20 \%$ & 1,4 & 5,1 & 1,7 & 4,6 & 0,8 & 2,1 & 1.5 & 6,3 \\
\hline & $\mathrm{E}-20 \%$ & 1,4 & 5,1 & 1,7 & 4,5 & 0,8 & 2,1 & 1,4 & 6,2 \\
\hline \multirow{2}{*}{$\frac{\overrightarrow{\mathrm{g}}}{\mathrm{E}}$} & $\alpha_{0}+1^{\circ}$ & 1,4 & 5,1 & 1,5 & 4,2 & 0,8 & 2,1 & 1,5 & 6,3 \\
\hline & $\infty_{0}-1^{\circ}$ & 1,4 & 5,1 & 1,8 & 49 & 0,8 & 2,1 & 1,4 & 6,2 \\
\hline \multirow{4}{*}{ 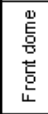 } & $\alpha+10^{\circ}$ & 2,1 & 54 & 0,1 & 21,3 & 1.8 & 4,8 & 4,5 & 20,4 \\
\hline & $\alpha-10^{\circ}$ & 1,5 & 5,1 & 3,1 & 65 & 0,6 & 0,7 & 0,3 & 0,6 \\
\hline & $\alpha+10 \%$ & 1,6 & 5,1 & 2,4 & 5,3 & 1,1 & 2,6 & 1,8 & 7,8 \\
\hline & $\alpha-10 \%$ & 1,3 & 5,1 & 1,7 & 4.6 & 0,7 & 1,6 & 1,2 & 5,0 \\
\hline \multirow{4}{*}{ 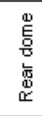 } & $\alpha+10^{\circ}$ & 2,4 & 10,1 & 4,0 & 143 & 1,2 & 2,1 & 3,3 & 20,6 \\
\hline & $\alpha-10^{\circ}$ & 1,3 & 4,8 & 3,7 & 140 & 0,8 & 2,1 & 2,4 & 10,5 \\
\hline & $\alpha+10 \%$ & 1,8 & 5,6 & 1,1 & 2,0 & 1,0 & 2,1 & 1,8 & 6,2 \\
\hline & $\alpha-10 \%$ & 1,0 & 4,2 & 2,6 & 8.7 & 0,7 & 2,1 & 1,8 & 6,2 \\
\hline \multirow{2}{*}{ 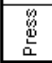 } & $10 \mathrm{bar}$ & 1,3 & 5,1 & 1,7 & 5,0 & 0,5 & 0,2 & 0,8 & 2,1 \\
\hline & 20 bar & 1,3 & 5,1 & 1,9 & 5,3 & 0,5 & 0,7 & 0,6 & 2,4 \\
\hline
\end{tabular}

The results of the multivariate analysis (Tab. 3) show, on the opposite of the analysis performed on individual parameters, that no significant modification has to be applied to the reference materials properties. The $1^{\text {st }}$ stage model can be optimized by a slight modification of the winding law which was initially approached by a theoretical law. For the $2^{\text {nd }}$ stage model, the best solution is essentially obtained through the use of a prior deformation of the model with a 18 bar internal pressure.

Table 3: $\quad$ Results of the multivariate analysis.

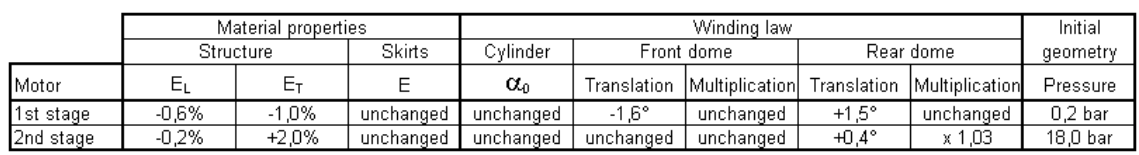

The verification carried out with this new set of input data confirms a significant improvement of the predictions for the internal pressure load case (Tab. 4), for the $2^{\text {nd }}$ stage model (Fig. 8b, d). For the $1^{\text {st }}$ stage model (Fig. 8a, c), the axial behaviour of the rear dome is much improved at the cost of a slight degradation of radial displacements prediction. The initial calculations for the compression load case (Fig. 7) are not affected by the modifications. 
Table 4: $\quad$ Comparative predictions for the internal pressure load case.

\begin{tabular}{|c|c|c|c|c|c|c|c|c|}
\cline { 2 - 8 } \multicolumn{1}{c|}{} & \multicolumn{4}{c|}{ 1st stage } & \multicolumn{4}{c|}{ 2nd stage } \\
\cline { 2 - 8 } \multicolumn{1}{c|}{} & Radial error (mm) & \multicolumn{2}{c|}{ Axial error (mm) } & \multicolumn{2}{c|}{ Radial error (mm) } & \multicolumn{2}{c|}{ Axial error (mm) } \\
\cline { 2 - 8 } \multicolumn{1}{c|}{} & Mean & Maximum & Mean & Maximum & Mean & Maximum & Mean & Maximum \\
\hline Reference & 1,4 & 5,1 & 1,7 & 4,6 & 0,8 & 2,1 & 1,4 & 6,2 \\
\hline Optimized & 1,5 & 5,3 & $\mathbf{1 , 1}$ & 2,0 & $\mathbf{0 , 6}$ & $\mathbf{0 , 9}$ & $\mathbf{0 , 3}$ & $\mathbf{1 , 3}$ \\
\hline
\end{tabular}

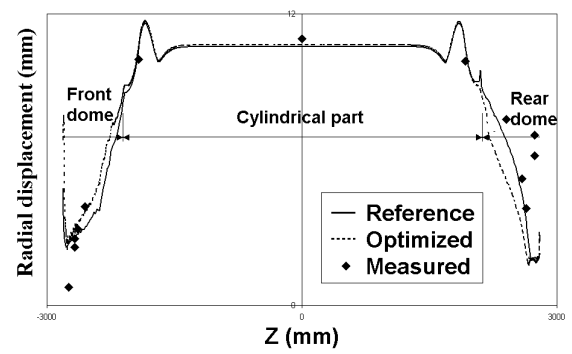

a) First stage

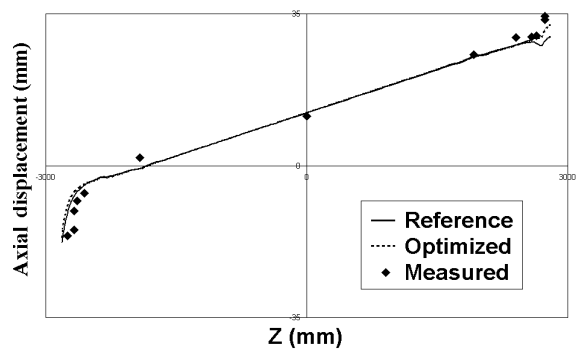

c) First stage

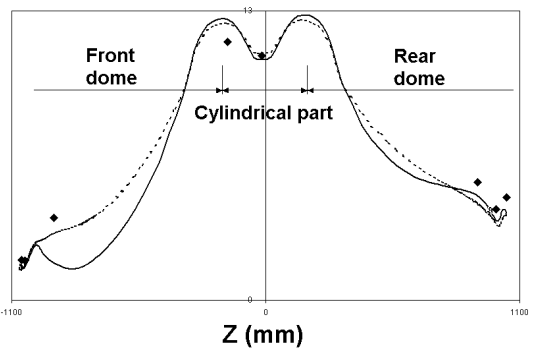

b) Second stage

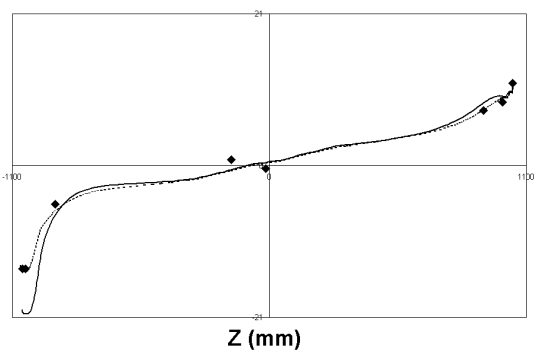

d) Second stage

Figure 8: $\quad$ Evaluation of the models for the internal pressure load case.

\section{Conclusion}

This study shows the potential interest of multivariate analyses for the development of mechanical models for structures with uncertainties concerning the input data (difficult characterization of filament wound materials, influence of the fabrication and qualification processes, unknown winding parameters, etc.). The method presented here for two structures and two load cases results in a considerable improvement of the predictions with a coherent modification of the input data. This procedure can be broadened to a multiple load case analysis with non-linear fits for each parameter. 